\title{
Interactive visual imagery and memory search for words and pictures
}

\author{
STEPHEN M. KERST \\ University of Wisconsin, Madison, Wisconsin 53706
}

\begin{abstract}
In a short-term recognition memory experiment with words, subjects: (1) subvocally rehearsed the words, (2) generated a separate visual image for each word, (3) generated an interactive scene with such images, or (4) composed a covert sentence using the words in the memory set. Contrary to Seamon's (1972) results in a similar study, a serial memory search was found in all conditions, instead of the simultaneous scan which was expected when items were combined in interactive images. In a second study with pictures as stimuli, subjects who generated imaginal interactions between separate pictures, viewed interacting pictures, or viewed separate pictures also showed a serial search, i.e., longer RTs were obtained when more stimuli were held in memory. Since interactive imagery facilitated performance in an unexpected paired-associate task with memory set stimuli, one can argue that subjects actually processed or generated such interactions. It was suggested that memory search might not be simultaneous in tasks where the test stimulus constitutes only part of a memory image.
\end{abstract}

Instructions to generate pictorial interacting mental images from words or separate pictures in a pairedassociate (PA) task have been found to improve recall and recognition performance (Paivio, 1971; Reese, 1970). Furthermore, Bower (1970) showed that instructions to generate an interacting image for each pair of words to be learned facilitated learning, while instructions to form separate images of the referents of the stimulus and response terms did not. Apparently it is the production of an interaction, not merely the generation of images per se, that is the effective process here. It is not clear, however, what covert processes are going on when instructions to generate interacting visual images are given.

Seamon (1972) tested the proposition that instructions to use imagery as an encoding strategy could affect the way in which information was retrieved from shortterm memory (STM). When no special encoding instructions are given, short-term recognition memory involves an exhaustive serial search of the contents of immediate memory (Clifton \& Tash, 1973; Sternberg, 1966, 1969). In these experiments, subjects were shown small sets (one to six members) of words, numbers, or nonsymbolic stimuli such as nonsense shapes or photo-

This research was sponsored by the Wisconsin Research and Development Center for Cognitive Learning, supported in part as a Research and Development Center by funds from the U.S. Office of Education, Department of Health, Education, and Welfare, Center Contract NE-C-00-3-0065. This study was conducted as a partial requirement for the author's doctoral degree. I am grateful to Joel R. Levin for supervising the dissertation and for helpful comments on this manuscript. Thanks are also due Robert E. Davidson and Steven $R$. Yussen for valuable suggestions during the planning stages of these experiments. Requests for reprints should be sent to Stephen M. Kerst, who is now at the Boys Town Center for the Study of Youth Development, The Catholic University of America, Washington, D.C. 20064. graphs of faces. After a retention interval of 2 or $3 \mathrm{sec}$, a test stimulus was presented, and subjects were asked to indicate whether the test stimulus was a member of the original memory set. For all types of materials, reaction time (RT) increased as a linear function of the number of items held in STM, with no difference between the slopes for positive and negative responses. The slope for "yes" responses would be expected to be half of the slope for "no" responses if the memory search was terminated when a match was found, since on the average only half of the items would have to be searched before a match was found for a positive test stimulus, while all items would have to be checked before a negative response could be made. The obtained results suggest that all stimuli in the memory set were serially compared to the test stimulus before either a positive or negative response was made. In such a recognition task, STM search appears to be an exhaustive rather than a self-terminating process.

Seamon (1972) predicted that if items in STM were encoded within one interactive image, a simultaneous comparison process would result instead of a serial one. In that study, independent groups of subjects were shown stimulus sets of one to three concrete nouns. The subjects were instructed to repeat the word subvocally (nonimagery condition), to generate a separate image of the referent of each word (separate imagery condition), or to generate one image which involved the referents of each word in an interactive scene (relational imagery condition).

In the nonimagery and separate imagery conditions, RT was found to increase linearly with the size of the stimulus set, with similar slopes for both "yes" and "no" responses. In the relational imagery condition, however, the size of the set did not affect the amount of time needed to indicate whether the test stimulus was 
a member of the original set. Apparently, the test stimulus was compared to all the memory set items one at a time in the first two conditions, while all stimuli in STM could be compared to the test stimulus simultaneously in the relational imagery condition. Seamon (1972) argued from these results that relational imagery instructions yielded a visual representation where items in STM were temporally and spatially united.

It is not clear that Seamon's (1972) findings are due to the generation of interacting mental images instead of the generation of interactions of unspecified modality. Like relational imagery instructions, instructions to generate sentences using the terms to be learned in a PA task improve performance (Paivio, 1971). Paivio argues that both verbal and visual codes may be available to the subject in some situations. The question that arises is whether the effects of relational instructions on memory search are found only in the case where visual imagery is presumed to relate or unite the items in the memory set, or whether instructions to generate a sentence would result in simultaneous access to the items involved in a sentence held in STM.

On the other hand, if a sentence generation instruction condition was found to involve serial comparisons, one could argue that interaction imagery and sentence generation instructions induce different kinds of retrieval (and perhaps storage) processes. In this case, the scanning of a generated sentence for a particular word could be characterized as a sequential word-byword (or phrase-by-phrase or piece-by-piece) process, while the scanning of a generated image could be likened to the viewing of a picture, where information is available more or less simultaneously.

Rothstein and Atkinson (1975) ${ }^{1}$, however, recently reported that they had failed to replicate Seamon's findings even when the rated vividness of the interactive scene was considered and the importance of using the scene as the basis of comparison with the test stimulus was stressed. The present experiments differ from this study in four ways which contribute to a more satisfactory test of the hypothesis that interactive relational imagery yields a simultaneous scan of a visual representation in STM. First, a sentence generation condition was included to determine if a simultaneous scan could be induced by verbal mediation instructions. Second, an extra interaction imagery group was run with the additional requirement that the images within the interactive scene generated by the subject had to be spatially united, i.e., touching each other. Third, actual drawings of both separate and interacting objects were used as stimuli in Experiment II in an attempt to provide clear cases of interactive visual imagery which were either supplied by the experimenter or generated by the subject from pictorial stimuli. Fourth, an incidental PA learning task with memory set stimuli was used to determine if the interactions were actually processed or generated in Experiment II. If imagery subjects actually used mnemonic interactions, they would be expected to outperform subjects who simply viewed the memory set stimuli as separate pictures without receiving instructions to combine them in an interactive scene.

Experiment II differs from a recent study (Klatzky \& Thompson, 1975) ${ }^{2}$ which also dealt with the process of the integration of whole stimuli from separately presented parts. These authors found that subjects did not integrate separately presented drawings of facial features when they were asked to determine if the features were identical to those in a second face-like stimulus. In Experiment II a different sort of integrated representation is investigated. In this situation, objects in a compound scene are connected by an active or spatial relationship of the kind that is known to "unite" the objects in terms of PA learning (Paivio, 1971), e.g., a scene where a $\log$ is rolling after a man. It is the effect of such relationships on the nature of STM search that is of interest in the present studies.

Experiment I was performed in an attempt to replicate Seamon's (1972) results and to determine if sentence and imagery coding strategies have different effects on the STM search.

\section{EXPERIMENT I}

\section{Method}

Subjects. Fifty students (25 males, 25 females) from the University of Wisconsin, Madison, were paid $\$ 2$ each to serve as subjects. All subjects had just served in a short experiment (15 min) concerning verbal and motor reaction times to the onset of a stimulus light.

Design. Five groups of 10 subjects apiece were tested. Each group received one of four instructional strategies: (1) subvocal repetition, (2) separation imagery, (3) interaction imagery, and (4) sentence generation. An additional interaction imagery group (5) was run with modified instructions after the first four conditions had been completed and the results had been analyzed. Data from this group were analyzed separately. Conditions 1 , 2 , and 3 provide for a replication of Seamon's (1972) study, while Conditions 4 and 5 are additions.

Memory set size was varied within subjects in a mixed-list format. A varied-set procedure was used where a memory set composed of new items was presented for every trial. Negative test stimuli were always new items that had not been shown before. Subjects were randomly assigned to conditions in blocks of four, where each block constituted a replication of the experiment.

Procedure. Sets of one, two, or three printed English concrete nouns similar to those used by Seamon (1972) were presented to subjects in horizontal arrays via slide projector cued by inaudible signals from a tape recorder. Stimuli in the memory set were presented simultaneously for 5 sec in Set Size 1, $10 \mathrm{sec}$ in Set Size 2, and $15 \mathrm{sec}$ in Set Size 3. The stimulus set was followed $3 \mathrm{sec}$ later by an auditory warning signal (a "click" from the projector) and a probe word $1 \mathrm{sec}$ after the click. The probe word remained visible for $7 \mathrm{sec}$. Three blocks of 18 trials were presented. Each block contained six trials (three "yes" and three "no" trials) for each of the three memory set sizes. The order of presentation of blocks was counterbalanced across subjects and the first block was considered practice. New words varying in length from three to nine letters were used on each of the trials, which were separated by intertrial intervals of $5 \mathrm{sec}$. For "yes" responses, each location on the screen (left, middle, right) of the probe word in the memory set appeared equally often. 
Stimuli fell into three classes: (1) potential actors or subjects of an active declarative sentence, (2) likely receivers of action or direct objects, and (3) probable objects of a prepositional phrase which could specify how or where the action was carried out. The words in a set were selected so that they could be combined to form sentences (e.g., "cowboy, car, barn;" "scissors, hose, jug;" "cat, apple," "knife, hammer;" "trench, glove, rake"). Each class of word was shown equally of ten in each location on the screen for Set Size 3, while actors and objects appeared on the left and the right sides of the screen with equal frequency for Set Size 2. Reaction times between the onset of the probe word and the subjects' vocal "yes" and "no" responses were measured by an electric timer (accurate to $.01 \mathrm{sec}$, synchronized with the projector, which was stopped by a signal from a voice-operated relay.

In the subvocal repetition condition, subjects were instructed to rehearse the memory set stimuli when they were presented and during the 3 -sec retention interval which preceded the probe. Subjects in the separation imagery condition were told to generate a separate image of a referent of each stimulus word and to keep the images spatially separate and noninteracting, while thinking about each in succession until the probe word was presented. In the original interaction imagery condition, subjects were instructed to form an image for each of the stimuli, to combine these images into one interactive scene (in cases where there was more than one stimulus), and to concentrate on the imaginal scene until the probe word was presented. Instructions to subjects in the new interaction imagery condition were modified to emphasize that the images in the scene should be touching each other. Examples of such images were shown, and the use of the interacting scene as the basis for judging the test word was stressed. In the sentence generation condition, subjects were asked to form sentences which described an interaction among the stimuli. When three words appeared on the screen, subjects were told to incorporate them into a sentence which described one thing acting on another. The remaining word was to be used to tell how or where the action was accomplished (e.g., "The cowboy drove the car into the barn."). For memory sets of two words, subjects were told to generate a sentence where one object was acting on the other (e.g., "The bus hit the tree."). When only a single word was presented in the stimulus set, subjects were told to rehearse it subvocally. Subjects were told to notify the experimenter if they were unable to comply with the imagery or sentence instructions on a particular trial rather than making a "yes" or "no" response. Examples were provided to subjects to make sure the task and instructions were understood.

\section{Results}

Trials where yes/no response errors were made were excluded from analysis. One such error occurred in each condition except the separation imagery group. Reported failures to generate images or sentences were likewise infrequent (only two in the interaction imagery condition) and were also excluded. Two subjects in the separation imagery condition who reported using interaction imagery were replaced.

The main hypothesis concerning the simultaneous or serial nature of the STM search process under different instructions was tested in two ways. First, following Seamon's (1972) procedure, linear trend comparisons based on the combined data from "yes" and "no" responses were conducted to evaluate the effects of memory set size on RT. Second, separate slopes were calculated for "yes" and "no" responses, and these values were tested to determine if they differed from zero. Additional comparisons were made within groups between slopes for "yes" and "no" responses in order to compare these results with the usual findings in the Sternberg (1969) paradigm. Comparisons were also made between groups for slope and intercepts to determine if instructions affected the encoding stage (intercept) and search stage (slope) of the task differently.

For the linear trend analysis, a median score for each set size and response type in each block was computed for each subject. These medians were averaged across blocks and "yes" and "no" responses at each memory set size (see Figure 1 for graphic representations of these averages and for least-squares linear functions for RT as a function of set size). In all conditions, linear trend comparisons revealed significant effects $(p<.05$, onetailed test) of set size on RT: subvocal repetition, $t(8)=9.95$; separation imagery, $t(8)=11.72$; original interaction imagery, $\mathrm{t}(8)=3.46$; sentence, $\mathrm{t}(8)=6.87$; and new interaction imagery, $t(8)=4.00$. The error variance associated with a trend contrast was computed separately for each condition because inspection suggested that the variances were not homogeneous across groups (i.e., a sevenfold difference between the largest and the smallest values), and since set size effects within conditions were of interest.

In an auxiliary analysis, the slope and intercept for RT as a function of set size were computed for each subject using all "yes" and "no" responses for Set Sizes 2 and 3. In order to make the present analysis comparable to that of Seamon (1972), "yes" and "no" responses for Set Size 1 were not considered. Seamon apparently intended to obtain estimates of the slopes for "yes" and "no" responses which were not affected by the longer latencies for "no" responses typically found at Set Size 1 (Chase \& Calfee, 1969).

Individual subjects' slopes and intercepts were then averaged within conditions to yield a mean slope and intercept for each group for "yes" and for "no" responses. "Yes" and "no" slopes in all five conditions were tested separately to determine if they differed from zero. All slopes showed such a difference except the "yes" slope in the original interaction imagery condition ( $p<.05$, one-tailed test). For "yes" slopes, values for $t(8)$ are as follows: subvocal repetition, 3.79 ; separation imagery, 3.86; original interaction imagery, 1.22; sentence, 4.00; and new interaction imagery, 2.96. For "no" slopes, values for $t(8)$ are listed: subvocal repetition, 3.37; separation imagery, 2.23; original interaction imagery, 2.94; sentence, 5.00; and new interaction imagery, 2.83 .

Using the slopes and intercepts from the four original conditions, an ANOVA was performed, with conditions as the independent variable and sex as a control variable. No significant differences for "no" slopes were found across conditions $[F(3,32)=1.19, p>.05]$. Slopes for "yes" responses differed significantly across conditions $[F(3,32)=2.92, p<.05]$, although no Tukey post hoc pairwise comparisons were significant at $\alpha=.05$.

Analysis of the intercept data for the four original 


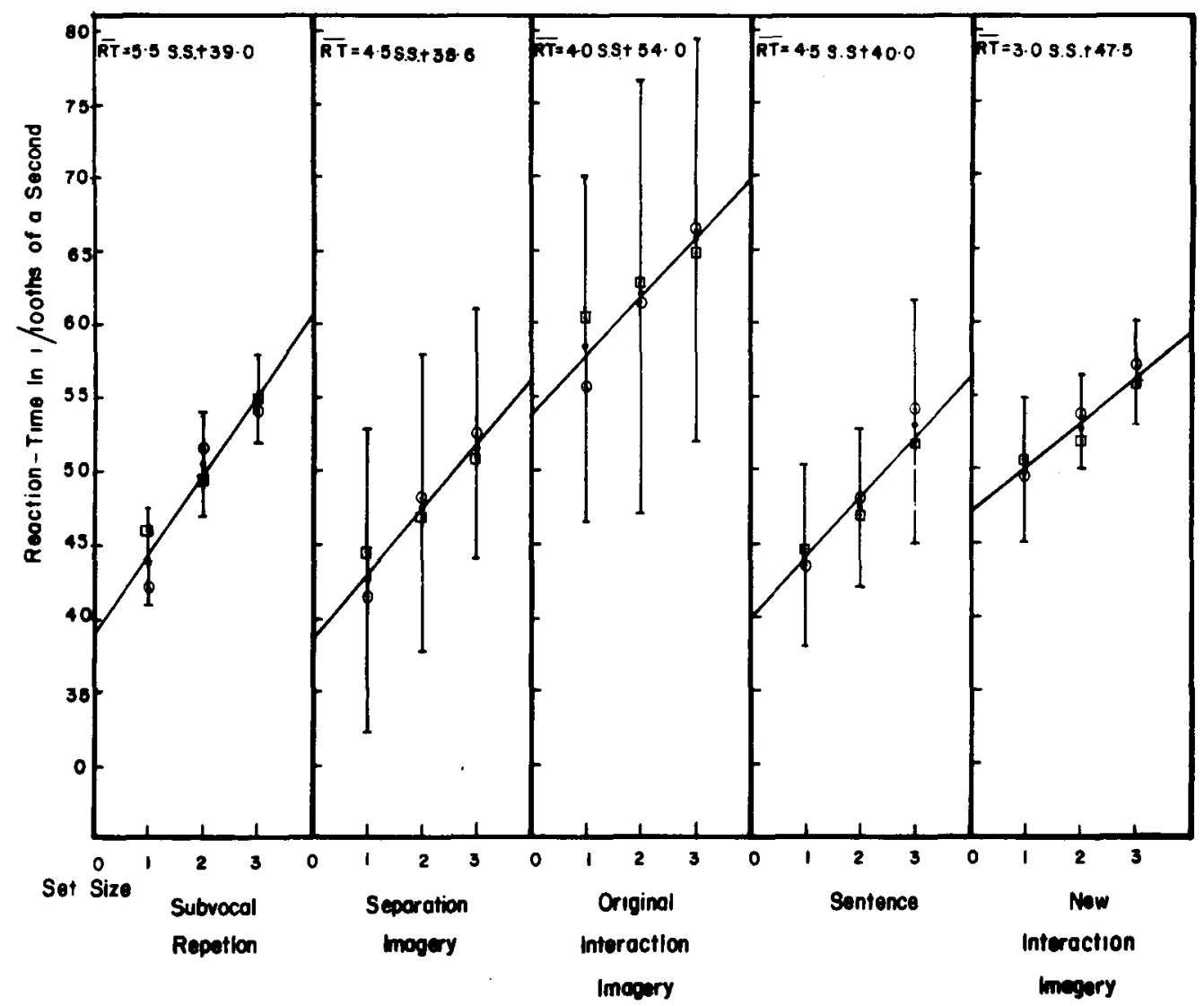

Figure 1. The relationship between RT and memory set size for each of the five experimental conditions. Mean latencies for positive responses (open circles) and negative responses (open squares) are shown. Overall means for each set size based on both positive and negative responses (solid circles) are shown with $\pm 1 \sigma$. Least-squares linear functions were applied to the overall means for each condition.

groups showed differences across conditions for "yes" responses $[F(3,32)=8.27, \mathrm{p}<.05]$ and for "no" responses $[F(3,32)=2.95, p<.05]$. Tukey pairwise post hoc comparisons at $\alpha=.05$ indicated that for "yes" responses the intercept for the original interaction imagery groups was larger than those of the repetition, separation imagery, and sentence conditions. For "no" responses, the original interaction imagery group displayed a greater intercept than the sentence group.

Slopes for "yes" responses were compared to those for "no" responses within all five conditions. Onesample $t$ tests revealed no yes/no slope differences in any condition ( $\mathrm{p}>.05$, two-tailed).

\section{Discussion}

Contrary to Seamon's (1972) results, interaction imagery instructions did not cause a simultaneous search of STM in the present experiment, even when subjects were told that the imagined objects must be in contact with each other and that the interactive scene should be compared directly with the test stimulus. In all conditions, significant linear trends were found for RT as a function of memory set size. Additional items in memory required extra time to be scanned, regardless of instructions given to subjects.

There were no differences in slope due to conditions, and there were no cases where "no" slopes exceeded "yes" slopes. These findings support Sternberg's (1969) view of the memory scan as an exhaustive serial process with a rate of $38.50 \mathrm{msec} / \mathrm{item}$. The one instance of a slope of zero occurred for "yes" responses in the original imagery condition, but was not replicated with either response type in the new interaction imagery group. Since an interaction imagery process would be expected to yield a simultaneous scan (zero slope) for both "yes" and "no" responses, the one instance of a zero slope cannot be taken as support for Seamon's (1972) hypothesis. The original interaction imagery group did display significantly higher intercepts than other conditions, which could indicate that interaction imagery instructions cause additional processes which precede the memory scan. These high intercepts must be interpreted with caution, since they were not replicated (descriptively speaking) in the new interaction imagery group, which performed more like the control group than like the original interaction imagery condition.

The results of Experiment I are similar to those of Rothstein and Atkinson (1975), who recently reported that they had failed to replicate Seamon's (1972) findings. Since interactive visual imagery failed to yield the expected simultaneous search of STM, the expected imagery effects can neither be contrasted with those of 
sentence generation nor explained in terms of them, as was originally anticipated.

A closer examination of the task indicates that even if a relational imagery strategy caused a simultaneous scan of items in STM, this effect might be concealed due to the fact that subjects presumably had to make some type of translation or modality switch between generated visual images held in memory and the printed words used as test stimuli. Chase and Calfee (1969) and Swanson, Johnsen, and Briggs (1972) found that such translations (switching from an auditory presentation of a word to a visual test stimulus and the reverse in the first case; switching from a nonsense shape to an associated nonsense label and the reverse in the second) increased the slope of the RT function in the Sternberg (1969) task. This finding is congruent with the proposition that each comparison of a memory representation with a test stimulus includes the additional time needed to translate the memory representation into the mode of the test stimulus for comparison. If, on the other hand, the test stimulus was translated into the mode of the memory representation (an operation that would happen once in the memory scan regardless of memory set size), an increase in intercept, rather than slope, would be expected, since a constant translation time would have been added to RT regardless of set size. The point here is that the slope increases found by Chase and Calfee (1969) and Swanson et al. (1972) would tend to counteract any slope reduction or tendency toward simultaneous scan in Seamon's (1972) task. For this reason, a recognition task which involves a comparison between a presumed image held in STM and a word as a test stimulus does not provide a suitable test of the hypothesis that interactive imagery allows a simultaneous search of all objects in the image.

Generalizing from the Chase and Calfee (1969) and Swanson et al. (1972) data, one would expect a greater slope in the separation imagery condition than in the subvocal repetition condition in the present experiment. This difference should occur; since subjects in the imagery group would have had to translate between images in memory and presented test words, while subjects in the subvocal repetition condition would not have had to make this translation. Such a slope difference was not found in the present study or in Seamon's (1972) experiment. This finding suggests at least two possibilities: (1) Subjects did not generate images and (2) subjects generated images but they were not used as the basis of the memory search. The fact that words were used as test stimuli may have discouraged subjects from either generating images or using them as a basis of comparison with the test stimulus, since the imagery and translation processes may have been seen by subjects as conflicting with the basic instructions to "respond as quickly and accurately as possible."

\section{EXPERIMENT II}

In order to test the hypothesis that interactive relational imagery causes a simultaneous search through items in STM, it was necessary to eliminate the presumed image-word translation process and to encourage subjects to make use of a visual code. The present experiment included only pictorial materials. The effects on memory scan of depicted visual interactions between pictured objects (in the provided interaction condition) were compared with those of interactions generated covertly by subjects from spatially separate pictures of objects (in the subject-generated interaction condition). For example, a provided interaction might consist of a picture of a cat eating an apple or a knife cutting a hammer, where the two objects were in contact and one was acting on the other. In the subject-generated interaction condition, subjects were asked to visualize such an interacting scene when they were shown pictures where a cat and an apple or a knife and a hammer were presented as separate objects which were not interacting. $\bar{A}$ control condition was included in which subjects were told to hold the spatially separate pictures in memory.

To determine if subjects were complying in some way with the instructions to generate visual interactions, an unexpected PA task was administered on memory set items at the end of the experiment. Since it is known that such instructions improve even incidental associative learning (Rowe \& Paivio, 1971), superior PA performance could be taken as evidence that subjects were attempting to comply with instructions and were actually engaging in some strategy. It is the effects of this interaction strategy on the rate of memory scan which are of interest here.

\section{Method}

Subjects. Thirty graduate students (15 males, 15 females) from the University of Wisconsin, Madison, volunteered to serve as subjects in a 45 -min session.

Design. Three groups of 10 subjects apiece were compared: control, provided interaction, and subject-generated interaction. Memory set size was varied within subjects in a mixed-list format.

Procedure. The subjects were tested individually. Sets of one or two line drawings of common objects (e.g., a house, a cat, a truck, a ball) were presented by a slide projector cued by inaudible signals from a tape recorder. Stimuli for Set Size 1 and all test items were centered in 2 by $2(35-\mathrm{mm})$ slides. Set Size 2 stimuli in the control and subject-generated interaction conditions were positioned symmetrically about the midpoint of the slide and the depicted interactions between objects for Set Size 2 in the provided interaction condition were centered in the slide frame. In cases where the test stimulus matched a presented item in the memory set, both were in the same orientation. This was done since RT measures have been found to be sensitive to orientation changes between pictures presented at the acquisition and test phases of a recognition task (Frost, 1972).

Although it would have been desirable to have included pictures for Set Size 3, materials were unavailable. The hypo- 
thesis that all items in an interactive image can be scanned simultaneously yields the prediction that memory set size will not affect the time required for the memory search. A difference in RT between Set Sizes 1 and 2 would be inconsistent with this hypothesis, while a failure to find such a difference would not provide strong support for the hypothesis unless similar results were found with Set Size 3 items.

As in Experiment I, memory set stimuli were presented for $5 \mathrm{sec}$ for Set Size 1 and $10 \mathrm{sec}$ for Set Size 2 in all conditions. A varied set procedure was used where a memory set composed of new items was presented for every trial. Negative test stimuli were always new items which had not been shown before. The memory set was followed by a 3-sec blank interval and an auditory warning signal, a test stimulus of $7-\sec$ duration $1 \mathrm{sec}$ after the signal, and, finally, by an intertrial interval of 5 sec. The subjects were instructed to say "yes" if the test stimulus was included in the memory set and to say "no" if it was not. "Yes" and "no" responses were equally likely, and each of the two serial positions for the target picture for "yes" responses in Set Size 2 was equally frequent. Reaction times between the onset of the test stimulus and the subjects' vocal responses were measured by an electric timer (accurate to $.01 \mathrm{sec}$ ) synchronized with the projector, which was stopped by a signal from a voice-operated relay. A preliminary block of nine trials was considered practice. Two blocks of 20 trials each (counterbalanced for order across subjects) yielded 10 "yes" and 10 "no" items at each memory set size.

In the control and provided interaction conditions, subjects were told to fixate visually on a dot centered on the screen while attempting to maintain a visual image of the stimulus picture(s) during the 3-sec retention interval. Subjects in the subject-generated interaction condition were asked to imagine a visual interaction between the two stimuli presented side by side for Set Size 2 and to center this interaction on the dot. Examples of pictorial interactions where two objects were touching and physically interacting in some way were shown. Subjects were told to indicate at the time if they failed to construct an interaction on a particular trial. All subjects received four "example" trials to clarify the nature of the task. An unexpected 20-item self-paced PA recall task, where the names of the left-hand pictures from Set Size 2 items were used as stimuli, was given at the conclusion of the experiment. In this task, the experimenter read the name of the picture to the subject, who attempted to respond with the name of the picture which had been presented with it in an item of Set Size 2.

\section{Results}

A median score for each set size and response type in each block was computed for each subject. On several trials, subjects were apparently inattentive and occasionally yielded an extremely long response latency. Outlying scores, which would have doubled the range within a block of trials if they had been included, were discarded. Relatively few scores had to be deleted in this manner: three for the control condition, eight for the provided interaction condition, and four for the subject-generated interaction condition, out of a grand total of 1,200 responses. One subject who indicated that he did not understand the instructions after completing the first block of trials was replaced. No yes/no response errors were made, and only one case of failure to generate an interaction was reported.

For all conditions, additional items in STM increased the duration of the memory search. Since set size effects within groups were of interest, rather than differences between groups, one-sample $t$ tests were performed on RTs for Set Sizes 1 and 2 in each group. Significant differences in RT due to set size were found in all groups $(\mathrm{p}<.05$, one-tailed test). For the control group, $t(9)=4.32$; for the provided interaction group, $t(9)=$ 2.94; and for the subject-generated instruction group, $t(9)=8.78$. Mean RTs for each set size and condition are shown in Table 1.

In the incidental test of PA learning of Set Size 2 stimulus pairs, both the provided and subject-generated interaction groups were superior to the control group [p $<.05$ for Tukey post hoc comparisons; $F(2,24)=$ $16.7, p<.05$ for overall test in a one-way ANOVA]. The mean number of correct responses in the control, provided interaction, and subject-generated interaction conditions was $4.5,12.5$, and 12.5 , respectively.

\section{Discussion}

The superior PA performance of the subjectgenerated interaction group relative to the control condition indicates that subjects in the former group complied with the interaction instructions in the intended way throughout the experimental session, since such instructions normally improve learning in a standard PA task. The facilitative effects of provided visual interactions obtained in the present experiment are also congruent with findings in the conventional PA paradigm.

Contrary to predictions based on Seamon's (1972) hypothesis, a simultaneous scan of the contents of STM is not produced by either a subject-generated or depicted visual interaction between pictorial items in the memory set. The present data, based on memory Set Sizes 1 and 2, indicate that the more items that are held in STM, the longer it takes to search through them, even when items are presented as combined interacting pictures or when subjects are instructed to mentally combine separate pictures in an interactive mental image. For all conditions, RTs for Set Size 2 were greater than those for Set Size 1, indicating a sequential rather than simultaneous search through items held in STM. This method of analysis, where group means for "yes" and "no" responses are combined at each set size, is identical to Seamon's (1972) procedure. The present memory scan rates were comparable to the figures of $38.56 \mathrm{msec} / \mathrm{item}$ obtained by Sternberg (1969) with digits and shapes.

Table 1

Means and Standard Deviations of Reaction Times (1/100ths of a Sec) for Each Set Size and Condition: Experiment II

\begin{tabular}{|c|c|c|c|c|c|c|}
\hline \multirow{3}{*}{$\begin{array}{c}\text { Set } \\
\text { Size }\end{array}$} & \multicolumn{6}{|c|}{ Condition } \\
\hline & \multicolumn{2}{|c|}{ Control } & \multicolumn{2}{|c|}{$\begin{array}{c}\text { Provided } \\
\text { Interaction }\end{array}$} & \multicolumn{2}{|c|}{$\begin{array}{l}\text { Subject- } \\
\text { Generated } \\
\text { Interaction }\end{array}$} \\
\hline & Mean & SD & Mean & SD & Mean & SD \\
\hline $\begin{array}{l}1 \\
2\end{array}$ & $\begin{array}{l}43 \\
46\end{array}$ & $\begin{array}{l}6 \\
5\end{array}$ & $\begin{array}{l}53 \\
57\end{array}$ & $\begin{array}{r}11 \\
8\end{array}$ & $\begin{array}{l}43 \\
49\end{array}$ & $\begin{array}{l}8 \\
8\end{array}$ \\
\hline
\end{tabular}


Seamon's relational imagery group showed a nonsignificant increase in RT of about $.015 \mathrm{sec}$, or $1 / 4 \sigma$, from Set Size 1 to Set Size 2, while no increase in RT was found with Set Size 3. In the present study, significant increases in RT between Set Sizes 1 and 2, which are of roughly two and three times the value found by Seamon, were obtained in the provided interaction condition $(.04 \mathrm{sec}$, or about $1 / 20)$ and the subject-generated interaction condition $(.06 \mathrm{sec}$, or about $3 / 4 \sigma)$. These data are not consistent with the hypothesis that interactive relational imagery will yield a simultaneous memory search, and they are not consistent with Seamon's (1972) findings. Since deviations from the linearity of the increase in RT with memory set size are generally small compared to set size effects (Nickerson, 1972), the sizeable difference in RT between Set Sizes 1 and 2 in the present study suggests that RT would probably also increase at Set Size 3, in contrast to Seamon's (1972) results.

\section{GENERAL DISCUSSION}

In Experiment $I$ it was found that instructions to generate an interacting compound image for words held in STM did not cause a simultaneous memory search, as had been expected from Seamon's (1972) findings. When pictorial materials were supplied in Experiment II, a simultaneous search was not obtained, even when subjects were shown interacting pictures which comprised the memory set.

Although provided and subject-generated visual interactions somehow "unite" the stimulus and response terms in terms of PA recall, rate of STM scan is a measure which is not sensitive to this effect, since separate pictures were scanned at the same rate as interacting ones. Even if interacting pictures were retained in STM as a compound image, the memory search task used by Seamon (1972) and in the present study would not necessarily indicate a simultaneous scan of STM. It may be that when a single picture is used as a test stimulus, individual pictures, rather than interacting scenes, are treated as distinct units in the STM search. That is, an image of two interacting objects would have to be scanned one object at a time if the test stimulus was a picture of a single object. In this way, the memory representation and the external stimulus to which it is compared would be treated by the subject as units of complexity.

Partial support for this proposition comes from a study with verbal materials. When the items in memory and the test stimulus are of comparable complexity, there is evidence that STM search rates do reflect a certain type of unity among elements within an item in memory. Clifton and Tash (1973) found that the rate of memory search did not differ for letters, onesyllable words, and three-syllable words. This finding suggests that the memory representation of a word is not scanned sequentially as a string of discrete elements such as letters or phonemes, but that it is treated as some sort of integrated entity which is scanned at a rate which is independent of the number of elements it contains.

On the other hand, if words were used as in the memory set, while the subject was given a single letter as a test stimulus, the individual letter would be expected to affect the search rate, as if a serial process was taking place. This type of situation, where the test stimulus is some part of a "larger" representation held in STM, is found in Seamon's (1972) study and in the present experiment with visual materials. Under such conditions, a simultaneous scan of STM would not be obtained even if subject-generated or provided pictorial interactions were retained as compound visual images. For this reason, it is argued that the rate of memory search in the Sternberg (1969) paradigm as used here will not indicate whether items in STM are combined in interactive visual image when imagery instructions are given or when interacting pictures are supplied.

A more appropriate technique would be to use a similar task where the test stimulus was a compound interacting picture and the memory set stimuli were either subject-generated or provided pictorial interactions. In this way, the memory representation and the test stimulus would be comparable in terms of complexity. This task would yield a more direct test of the proposition that, if items are encoded within an interactive image in STM, they should be subject to a simultaneous memory search process.

Klatzky and Thompson (1975) recently used a similar task to determine whether subjects integrate separately presented drawings of facial features when they are asked to compare these features with a complete face. Under these conditions, where subjects are required to discriminate between different values of a feature (e.g., different types of noses), integration did not take place. The question remains whether such a task would reveal integration effects under conditions such as those in Experiment II, where the objects in a compound scene are connected by an active or spatial relationship, and where precise visual discrimination between similar features is not required.

\section{REFERENCES}

Bower. G. H. Analysis of a mnemonic device. American Scientist, 1970, 58, 496.510.

Chase. W. G.. \& Calfee. R. C. Modality and similarity effects in short-term recognition memory. Journal of Experimental Psychology'. 1969, 81, 510-514.

Clifton. C.. \& Tash, J. Effect of syllabic word length on memory search rate. Joumal of Experimental Psychology. 1973. 99. 231-235.

Frost. N. Encoding and retrieval in visual memory tasks. Journal of Experimental Psychology, 1972, 95, 317-326.

KLATZKY, R. L., \& ThOMPSON, A. Integration of features in comparing multifeature stimuli. Perception \& Psychophysics. 1975, 18, 428-432.

NiCKERSON. R. S. Binary-classification reaction-time: A review of some studies in information-processing capabilities. Psychonomic Monograph. Supplements. 1972, 4. Whole No. 65. 275-318. 
Paivio, A. Imugery and Verbal Processes, New York: Holt. Rinehart. \& Winston. 1971.

REESE. H. W. (Chm.). Imagery in children's learning: A symposium. Psychological Bulletin, 1970, 73. 383-421.

Rothstein. L. D.. \& Atrinson, R. C. Memory scanning in visual images. Memory \& Cognition, 1975, 3, 541-544.

Rowe. E. J.. \& Paivio, A. Imagery and repetition instructions in verbal discrimination and incidental paired-associate learning. Joumal of Verbal Learning and Verbal Behavior. 1971, 10, 668-672.

SEAMON. J. G. Imagery codes and human information retrieval. Journal of Experimental Psychology, 1972, 96, 468-470.

Sternberg. S. High speed scanning in human memory. Science, 1966. 153, 652-654.

Sternberg. S. Memory-scanning: Mental processes revealed by reaction-time experiments. American Scientist. 1969. 57. 421.457.
Swanson, J. M.. Johnsen. A. M.. \& Briggs. G. E. Recoding in a memory search task. Joumal of Experimenal Psychology. 1972. 93. 1.9.

\section{NOTES}

1. The experiments reported here were done without prior knowledge of the replication attempt by Rothstein and Atkinson (1975).

2. The present experiments were completed without knowledge of the related work by Klatzky and Thompson (1975).

(Received for publication December 1, 1975: revision accepted March 4, 1976.) 